\title{
Process Physics: Quantum Theories as Models of Complexity
}

\author{
Kirsty Kitto \\ The School of Chemistry, Physics and Earth Sciences, \\ Flinders University, Adelaide, Australia
}

April 30, 2007

\begin{abstract}
Originally based upon a pregeometric model of the Universe, Process Physics has now been formulated as far more general modelling paradigm that is capable of generating complex emergent behaviour. This article discusses the original relational model of Process Physics and the emergent hierarchical structure that it generates, linking the reason for this emergence to the historical basis of the model in quantum field theory. This historical connection is used to motivate a new interpretation of the general class of quantum theories as providing models of certain aspects of complex behaviour. A summary of this new realistic interpretation of quantum theory is presented and some applications of this viewpoint to the description of complex emergent behaviour are sketched out.
\end{abstract}

\section{Process Physics}

The dominant modelling methodology of physics is static. We model the universe as a $3+1$ dimensional pre-existing structure, where time shares a very similar status to that of space, despite our everyday experiences of the two phenomena as being remarkably different. Process Physics sprang out of an initial desire to explain from where such a structure could have emerged (i.e., to form a pregeometric model of the Universe). A particular aim was to explain the relationships between space, time and matter, especially with respect to how such phenomena could dynamically emerge from an underlying model. 
The original pregeometric modelling has proven to be, at least in its initial stages, rather successful [1], and the reader is encouraged to consult that reference for more details. This paper will take a different approach. It is becoming apparent that the dominant modelling methodology of physics is not isolated and this has led to a search for new approaches to modelling in general [2]. This paper will discuss some of the work that has been performed during this search for a more process oriented approach to modelling.

The standard modelling paradigm of science is dominated by reductionism. This technique, which involves breaking an apparently complex problem into smaller, more manageable pieces, reduction and then combining the solutions obtained from these smaller problems into a larger solution which represents the original system, synthesis, has been remarkably successful in science. However it appears likely that this technique is approaching the end of its general scientific validity [2,3,4]. Many of the systems in which we are now interested cannot be so simply approached, a problem which has led to a general resurgence of holistic and systems oriented approaches. ${ }^{1}$ There is a general pattern emerging from this work; most of these problems of analysis tend to appear when an attempt is made to apply reductive techniques inappropriately to systems that are often classified as complex in some manner that is yet to be adequately defined [5]. Despite this problem of definition, a number of characteristics shared by such systems can be identified which make the appropriateness of their analysis by standard reductive approaches somewhat dubious. Such characteristics include:

1. Dynamic contingent behaviour indicative of a constant processing of information, energy, matter etc. This is in contrast to the generally very static models currently used where temporal processes are represented by mapping them to a real number line. Such a geometric approach to time tends to lose some of the most commonly experienced temporal phenomena, and has led to a number of well known problems in physics such as the lack of an arrow of time in our models, and the denial of a present moment [6].

2. Contextual responses to environmental perturbations, which mean that the system cannot be straightforwardly separated into a subsystem of interest and an environment that can be ignored [2].

3. Hierarchical organisation where generally stable subcomponents functioning on relatively fast timescales are incorporated (often nonadditively) into larger structures operating more slowly [7, 8]. A process of

\footnotetext{
${ }^{1}$ See for example the list of references at http://pespmc1.vub.ac.be/EVOCOPUB.html
} 
feedback is often manifested too, where the higher level of the hierarchical structure so formed can exert causal control over the lower level components [9].

4. Far from equilibrium and dissipative behaviour [10], which means that traditional models based upon static equilibria and other standard thermodynamic concepts are not necessarily applicable.

5. The exhibition of long range order, which is stable in the face of perturbations. ${ }^{2}$ This is an unusual feature, perturbations have traditionally been thought to disrupt long range order, and this is generally the case for classical systems, but not all systems are so unstable in such situations. For example, living organisms are not significantly disrupted by incoming energy, instead they convert such energy into chemical form and transport it over far greater distances than are predicted by linear models, leading to the so-called "bioenergetic crisis" [11].

6. Relational structure and evolution. The traditional modelling of such systems as objects exhibiting a number of interactions between themselves tends to limit the emergent behaviour that can be exhibited by them [12]. For example, in attempting to model the Universe using a number of fundamental objects we lose our ability to describe those very objects.

Interestingly, an analysis of the low level relational model of Process Physics (which will be discussed in the next section) reveals that it shares a number of these characteristics [6], a result that led to an indepth analysis of the reasons why this might be the case [2]. This paper will discuss some aspects of this journey of analysis. It will start with a quick review of the original low level relational model of Process Physics, illustrating the way in which it appears to be capable of generating interesting emergent behaviour. An analysis of the structures emergent from within this model will reveal that it is capable of dynamically generating apparently complex hierarchical behaviour. This will lead us to an examination of the historical roots of the model, which lie in quantum field theory (QFT), and to the hypothesis that quantum theories are capable of describing a far wider class of system than is generally considered to be the case. In particular, the claim will be made that QFT is capable of describing complex emergent behaviour. The remainder of this work will consist of a brief survey of some future promising avenues of research that follow from such a conclusion.

\footnotetext{
${ }^{2}$ At least within the limits of the systems tolerances. Outside this range catastrophic failure generally results.
} 


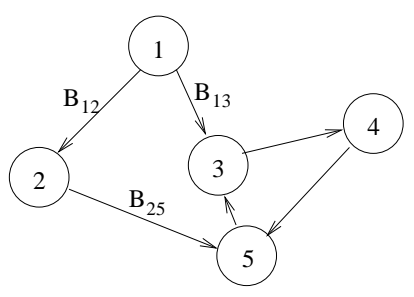

(a)

$$
\begin{aligned}
& \text { node } \\
& \begin{array}{ccccc}
1 & 2 & 3 & 4 & 5 \\
2 \\
3 \\
4 \\
4 \\
5
\end{array}\left(\begin{array}{ccccc}
0 & 1 & 1 & 0 & 0 \\
-1 & 0 & 0 & 0 & 1 \\
-1 & 0 & 0 & 0 & 1 \\
0 & 0 & -1 & 0 & 1 \\
0 & -1 & 1 & -1 & 0
\end{array}\right)=\left[\mathrm{B}_{\mathrm{ij}}\right]=\mathrm{B}
\end{aligned}
$$

(b)

Figure 1: A simple relational structure of nodes and their connections. (a)Nodes $i$ and $j$ are considered connected if they have a non-zero $B_{i j}$ value. Arrows indicate the sign of the $B_{i j}$ value. (b) This structure of nodes and their connections can be represented in an antisymmetric matrix, zero values represent no connection between nodes $i$ and $j$, while a nonzero value indicating a connection is represented here as \pm 1 depending upon the direction of the arrow drawn in (a).

\subsection{The Low Level Process Physics Model}

The initial low level model constructed within the Process Physics paradigm attempts to work around the static object driven methodology of reductionism by adopting a more relational approach. This is achieved by starting with a set of nodes for the sake of analysis, but instead of focussing upon the objects themselves, the connections between individual nodes are analysed. Thus, considering a set of $N$ nodes, we assume that they are connected in some way, with a connection strength between node $i$ and node $j$ given by the real value $B_{i j}$ (see figure 1 ). We represent a set of these relational values as a square antisymmetric ${ }^{3}$ matrix $B$. Notice that while the pitfalls of object driven methodologies have been explicitly recognised above, we are still driven to talk in terms of nodes; it is very difficult to leave an object-based methodology behind. This is due to the very nature of our analytic techniques which have always been based upon the reductionist methodology. However, any system simply positing such a priori objects cannot be considered fundamental when modelling the Universe, as the explanation of these objects must lie outside the system being modelled. A partial solution to this dilemma arises if we recognise that the nodes can in turn be defined for

\footnotetext{
${ }^{3}$ Antisymmetry ensures that $B_{i i}=0$ thus avoiding explicit node self connection. The internal structure of nodes will be incorporated shortly.
} 
the purposes of the model as a system of nodes. ${ }^{4}$ It becomes apparent that all nodes can be thought of as composed of collections of nodes in turn, and in particular that the start up nodes can be viewed as names for subnetworks of relations. This result is ensured if the constructed system exhibits selforganised criticality (SOC), which enforces a fractal structure on the system $[13,14]$.

The SOC requirement is intrinsically linked with a new processing notion of time in this system. This is because the relational fractal structure is generated by a noisy non-linear iterative map displaying SOC behaviour. Thus in attempting to construct a model that does not postulate a priori fundamental objects, we find the need to introduce a time-like process. In contrast, standard physics with its use of a priori objects is linked with the standard geometrical model of time and the associated problems mentioned above.

The particular map used was suggested by the Global Colour Model of quark physics [15]. Details of the history of this map will be discussed in section 4, but for now we simply assume that the $B$ matrix representing this system of nodes updates discreetly according to the following iterative process:

$$
B_{i j} \rightarrow B_{i j}-\alpha\left(B+B^{-1}\right)_{i j}+\omega_{i j},
$$

where $i, j=1,2 \ldots, N$ and $N \rightarrow \infty$. The constant $\alpha$ is an arbitrary tuning parameter [16], while the term $\omega_{i j}$ represents an additive noise term, which provides a sense of openness to the system.

At each iteration, the action of the noise term leads to the creation of new $B_{i j}$ links, incorporating a sense of innovation and contingency into the system. The noise term, when used iteratively in equation (1) is responsible for the notion of time that arises in the model. The dynamics are irreversible, with one particular past, which can be recorded as a history, but not relived. Future states of the system cannot be known. However certain sets of ensemble predictions can be made. In this sense a dynamical notion of time is captured by the system, with a markedly different ontology from the static four dimensional spacetime of standard physics. This leads to the identification of this system and its associated modelling techniques under the broad categorisation of Process Physics. We note for now that this modelling of time is far more appropriate in the modelling of living systems, providing a sense of contingency and dynamism that is generally lacking from more standard approaches.

The nonlinear matrix inversion term also performs a critical role in the system. It causes separate structures brought into existence by the noise

\footnotetext{
${ }^{4} B$ is assumed to be a very large $(\rightarrow \infty)$ matrix.
} 
term to link up, modelling a process of self-assembly. It is interesting to examine the dynamics of this process in detail.

The system can be started with $B \approx 0$ which represents the absence of any significant relational information. Under successive iterations of equation (1) the $B$ matrix assumes a sparse structure that can be organised into a block diagonal form. This suggests that there is a tendency for modes to organise into some sort of modular structure, a point that we shall return to in section 2.1 .

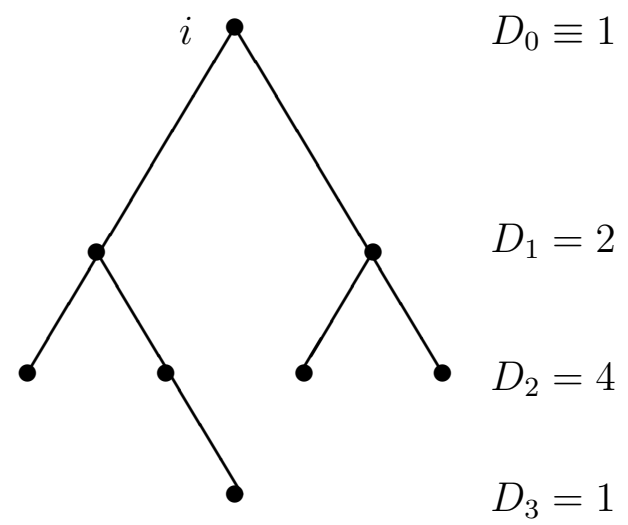

Figure 2: A $N=8$ spanning tree for a random graph (not shown) with $L=3$. The distance distribution $D_{k}$ is indicated for node $i$.

Assuming that the large $\omega_{i j}$ arise with fixed but very small probability $p$, the geometry of the structures formed can be revealed by studying the probability distribution of minimal spanning graphs with $D_{k}$ nodes and $k$ links from an arbitrary node $i$ where $D_{0} \equiv 1$ (see figure 2). This probability distribution is given by [17]

$$
\mathcal{P}[D, L, N] \propto \frac{p^{D_{1}}}{D_{1} ! D_{2} ! \ldots D_{L} !} \prod_{i=1}^{L-1}\left(q^{\sum_{j=0}^{i=1} D_{j}}\right)^{D_{i+1}}\left(1-q^{D_{i}}\right)^{D_{i+1}}
$$

where $q=1-p, N$ is the number of nodes and $L$ is the maximum depth from node $i$. The most likely pattern can be found by numerically maximising $\mathcal{P}[D, L, N]$ for fixed $N$ with respect to $L$ and $D_{k}$. This procedure has been performed [18], and the following results are from that analysis.

Figure 3 shows the set of $D_{k}$ (distance distribution) values obtained from one of these numerical experiments, where the log of the probability of a large noise value is set at $\log _{10} p=-6$, and the number of nodes is fixed at $N=5000$. Also shown in the figure is a curve

$$
D_{k} \propto \sin ^{d-1}(\pi k / L),
$$


with best fit to the data when $L=40$ and when the dimensionality of the fit, $d=3.16$. This same curve is obtained from the surface area of a $n$ dimensional sphere. Figure 4 shows the range in $d$ for fixed $N=5000$ and varying $p$ values. We see that for $p$ below some critical value $\log _{10} p<-5$, $d \approx 3$.

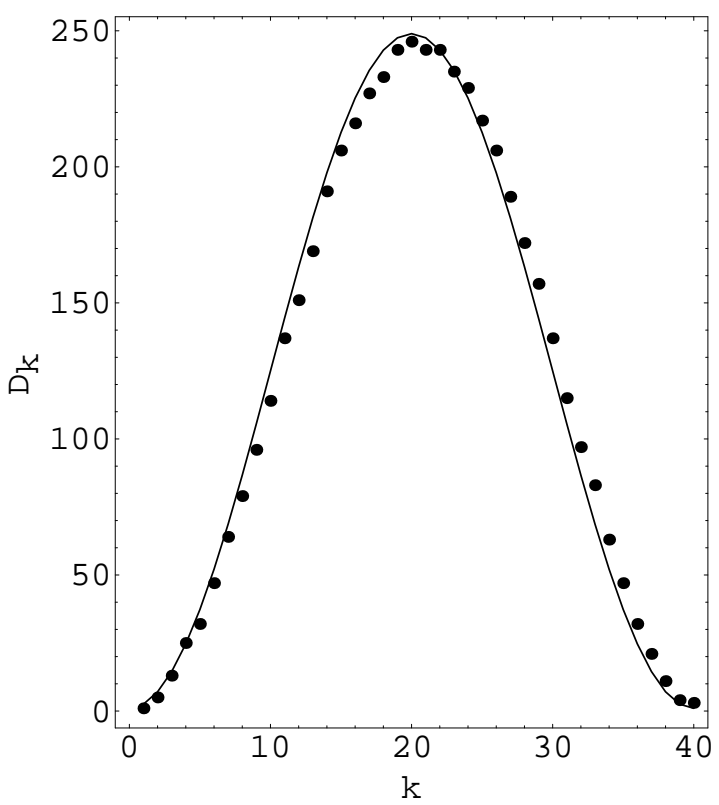

Figure 3: The distance distribution $D_{k}$ between nodes for a typical emergent structure under equation (1). This is obtained by numerically maximising $\mathcal{P}[D, L, N]$ where $N=5000$ and $\log _{10} p=-6$. A curve is also drawn showing that $D_{k} \propto \sin ^{d-1}(\pi k / L)$, with best fit $d=3.16$ and $L=40$.

This indicates that the connected nodes have a natural embedding in a $S^{3}$ hypersphere, which is very suggestive of the 3-dimensionality of space. Thus this model goes some way towards predicting the observed three dimensional structure of space as an emergent phenomenon, a feature that is usually assumed in physics. Notice that the nodes are not exactly embeddable (which would require $d=3$ ), there is a proportion of extra links. This is a key observation which forms the basis of a theory of matter as a quantum foam that is currently under development [1]. We shall discuss the underlying ideas of this theory shortly.

While they are not the only structures generated by the system, their maximum likelihood makes the hypersphere structures the most common. Splitting the large $B$ matrix into its constituent independent submatrices 


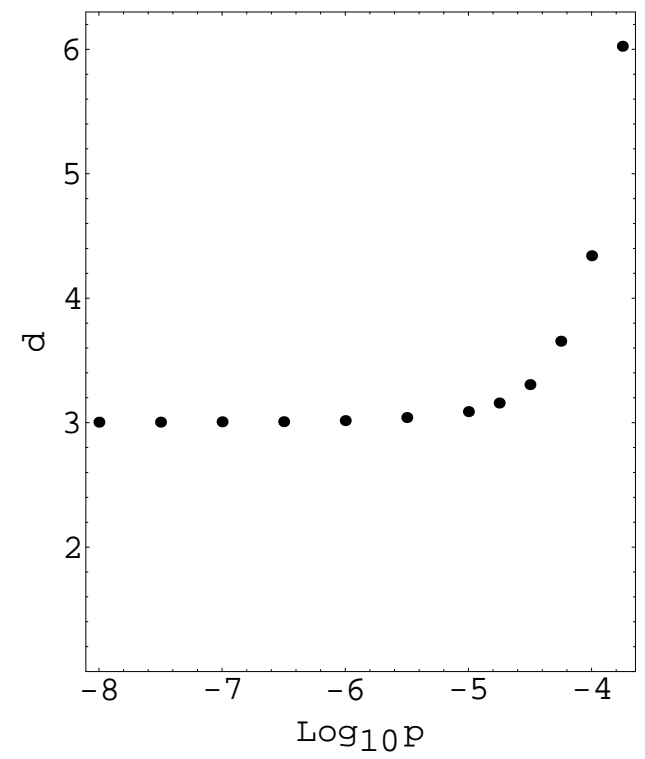

Figure 4: Range of $d$ values for $N=5000$ as a function of the probability $p$ of large noise values.

$B_{\text {sub }}$ we realise that each is almost $\operatorname{singular}\left(\operatorname{det}\left(B_{\text {tree }}\right) \approx 0\right)$ but that the noise term ensures extra $B_{i j}$ terms which lead to a small valued determinant. This small valued determinant implies that the next iterative step of the system will lead to new large valued $B_{i j}$ entries upon inversion of the $B$ matrix. Hence tree structures are sticky, at each iteration cross-links form between the structures which act to join them, and to produce larger structures. This behaviour has been examined in detail in a recent PhD thesis [16].

Thus, under the influence of the iterative equation (1) the system can be seen to 'grow' with a steady increase in relational structure. As the tree structures stick together, they become less easily embeddable in a $S^{3}$ structure. This phenomenon can be seen in figure 4, where beyond the critical value of $d \approx 3$ the dimension of the structures quickly becomes very high; they are no longer embeddable in $S^{3}$ and should instead be thought as defects in the system. These defect graphs gradually lose the ability to form new links, which tend to arise only at the level of 'leaves', or the endpoints of the graphs. As a result the defects become unable to sustain themselves and eventually they fade away from the system, or 'die'. However, the system itself generally grows faster than it loses such structures (see [16] for more details of these dynamics).

The nonlinear term in (1) is self-referencing; all elements of $B$ that arise in a previous iterative step are required in the computation of the next value 
of each $B_{i j}$ element. Thus, this term can be seen to incorporate a weak notion of internal self-observation into the system. In particular, any node has the capacity to profoundly affect the rest of the system if it randomly receives a large $\omega_{i j}$ value at the next iteration of the map. The fact that one node is not 'close' to another in a particular $S^{3}$ embedding does not mean that it cannot be affected strongly by it. This leads to a very strong form of contextual behaviour in the system, no one element can be considered as isolated, in fact, at the next iterative step it may become strongly linked to a node which was previously not considered important to its dynamics.

\section{Emergent structures within the Process Physics paradigm}

There is a sense of emergence in this system. Both the embeddable and the associated nonembeddable defect structures which appear to be forming are not explicitly present in either the update equation (1) or the original very simple relational structure of nodes and connections. An examination of this claim within the framework of hierarchy theory $[7,8,19,20,21,22,23]$ helps to clarify the form of emergent behaviour realised by this system.

\subsection{The hierarchical nature of the model}

An immediately apparent characteristic of this system is the block diagonal form of the matrix $B$ which arises under the influence of the update equation (1). This block diagonal form also arises in Simon's near-decomposable systems [22], making it possible to form an understanding of this system as a set of nearly-decomposable modules. This result makes sense. If nodes are seen as themselves made up of structures of connected nodes, then clearly some sort of modular hierarchical system has been created.

It is also possible to understand the relational model as forming at least a $2 n d$ order hyperstructure according to a theory of emergent hierarchical structure proposed by Baas [20]. This framework depends fundamentally upon the notion of observation used in the identification of new, higher order structures. Thus, in order to register the emergence of new structures or entities within a system it is necessary to identify a mechanism capable of observing those entities. Given that this system is aimed at modelling the Universe, we might ask what such a mechanism could be. How could an observational mechanism external to the Universe be identified? Such a mechanism can be found through the adoption of what might be regarded as an external perspective, but this does not imply that an entity exists externally 
to the Universe, merely that observation generally has to be implemented at a different modelling level. This point has been recognised by a number of different researchers $[24,25,26]$, many of whom claim that it is necessary to develop internal perspectives in order to understand complex phenomena such as the mind and life. The term endophysics [27, 28, 24] has been coined to refer to the study of systems which have observers enclosed within them. Such a view is participatory; how we look determines what we see, and with the development of new internal perspectives we might begin to understand the way in which the context of such an observer can affect the observations they make of the system itself [2]. Through a proper treatment of context we lose much of the confusion that often surrounds an examination of the Universe; an observer of the Universe can exist within that Universe. However, it will be necessary to adopt more than one model of the Universe in order to explain its complex emergent behaviour. We shall return to this point in section 3 .

To construct a Baas hierarchy, we consider the original nodes used in the construction of the relational structure to be a very simple family of $N$ firstorder structures $\Xi^{1}=\left\{\Xi_{r}^{1}: r=1,2, \ldots N\right\}$, where a first-order observational mechanism $O^{1}$ is defined as membership in the set $\Xi^{1}$. Now, under the influence of equation (1) which we represent as an update functional $R$, we find that these nodes join up, forming a second order structure:

$$
\Xi^{2}=R\left(\Xi_{i}^{1}, O^{1}, B_{i j}\right) \quad i, j=1,2, \ldots, N
$$

where $B_{i j}$ is the connection strength between nodes and $\Xi_{j}^{2}$ designates the structure that appears in the $B$ matrix in the previous sections. The family of second order structures consists of the maximum likelihood structures discussed above that are embeddable in the three-dimensional hypersphere $\left(E_{S^{3}}\right)$, along with the non-embeddable defect structures. Considering the space-type structures alone, we can define the property of being embeddable in $S^{3},\left(E_{S^{3}}\right)$ as a second order observationally emergent property,

$$
P_{E_{S^{3}}} \in O^{2}\left(\Xi^{2}\right) \text {. }
$$

That is, this property is not present in the simple set of nodes that we started with,

$$
P_{E_{S^{3}}} \notin O^{2}\left(\Xi^{1}\right)
$$

rather, it becomes apparent under the influence of the update functional (1).

The analysis of the remaining structure of this system is very complex and only in the preliminary stages, but it is expected that stable patterns identifiable as third order structures will emerge in the system. The full theory of this process is currently under development, but the general argument can be sketched out. 
We can somewhat artificially classify the structures in the system as being either exactly embeddable in $S^{3}$ (the $E_{S^{3}}$ structures), or non-embeddable structures which we will term defects $(D)$. It is expected that the system contains stable defects, which we shall term Topological Defects $(T D)$. The stability of these structures will be structural, and constitutes a new third order observation mechanism, $O^{3}$, within the system. Thus it is expected that the stable $T D$ 's will form a set of $K$ new emergent structures $\Xi^{3}=$ $\left\{\Xi_{v}^{3}: v=1,2, \ldots K\right\}$ within the system. Equation (1) ensures a constant updating of their components - rather like the units of an organism which are being constantly regenerated. These $T D$ 's (if rigorously identified) would be third order structures in the hierarchy. That is

$$
P_{T D} \in O^{3}\left(\Xi_{v}^{3}\right) \text {, and } P_{T D} \notin O^{3}\left(\Xi_{r}^{2}\right)
$$

because their emergent stability within the system is defined by their structural stability, i.e. it constitutes a new observation mechanism.

Thus, there is a sense of non-computational, or rather observational emergence (as defined by Baas [20]) in this system. More than one mode of analysis is required in order to understand its behaviour. This suggests that this system is truly exhibiting some sort of very complex behaviour [12], a concept that will be discussed more in the following section.

So far, a third order hyperstructure has been identified (but not rigorously at the third order).

$$
\text { nodes }>\underbrace{S^{3}+D}_{O^{2}\left(\Xi^{2}\right)}>\underbrace{\text { stable } T D}_{O^{3}\left(\Xi^{3}\right)}>\ldots
$$

It is expected that more structure will emerge from the system. This is because the identified stable structures of this model have a deep connection with the more standard object driven methodologies of particle physics. In [29] a link to the theory of preons ${ }^{5}$ was discovered, and therefore it appears that these systems can recover much of the behaviour of the Standard Model.

Despite this promise, the analysis of this system from this bottom up level is extremely challenging, both computationally and analytically. This problem has made it necessary to attempt a more top down understanding of this system [2]; why does it generate such interesting emergent behaviour?

One set of clues as to why this simple model is behaving in such a manner can be found by referring to the discussion from section 1. Summarising that discussion: this system was designed to be more relational than is normally the case, thus it avoids some of the most obvious traps of an object

\footnotetext{
${ }^{5}$ Preons are one of the proposed replacement 'fundamental particles' which in bound states form quarks $[30,31]$.
} 
driven modelling approach; it exhibits hierarchical behaviour; it has been designed to be far from equilibrium and dissipative; SOC behaviour is intrinsic to the model; there is a sense of long range order, even though most strongly connected nodes will tend to associate closely (i.e., embed well in a $3 D$ hypersphere) the defects associated with this model allow for long range connections between nodes, and some of these are expected to exhibit stability due to their topological nature. Perhaps most importantly, this system exhibits contextual responses; the dynamical, process-type understanding of time in this model means that different runs of the system, while exhibiting similar structure formation, will always exhibit contingent "once-off" events that will depend strongly upon both the random noise terms that arise at each iteration, as well as upon the pre-existing values of the $B_{i j}$.

Thus, there is every reason to suppose that this system is exhibiting some sort of complex emergent behaviour.

\section{$3 \quad$ High End Complexity}

The complex emergent behaviour realised by this simple relational system is not generally displayed by models of complex systems. For example, it is generally accepted that models capable of generating open ended evolution, or more than two levels of hierarchical behaviour have yet to be realised [32]. Yet there is every reason to suppose that such behaviour is being exhibited by this model (although this is very difficult to verify). Is the behaviour of this model complex? We must be careful with our conclusions. There is an ongoing debate in the complex systems science community about what exactly complex behaviour entails, and the search for a single unifying understanding of complexity may not in fact lead to one obvious definition $[5,33]$. It has recently been proposed that complexity should itself be regarded as a complex concept, with complex systems exhibiting a range of different behaviours and being appropriately modelled by a number of different techniques and concepts; [12], more details can be found there. In essence that discussion introduces a notion of high end complexity to describe those systems that cannot be modelled using our more standard reductive techniques; systems exhibiting high end complexity tend to require more than one mode of analysis for a complete understanding $[2,12]$. Such systems also display highly contextual responses to perturbations, which often means that how we analyse them can determine what we see. This suggests that the scientific ideal of an objective reality immune from our observations does not exist. However, it does not imply that reality is subjective. Generally, the contextual responses of such systems make existing models of their generation 
from presumably simpler subcomponents unsatisfactory, and their dynamics and evolution once created is not usually well understood either. We require new theoretical tools capable of properly incorporating such contextual dependency into our models. Is it possible that such tools might result from the Process Physics approach?

As an example of a system exhibiting high end complexity we might consider the process whereby a fertilised egg can divide, differentiate and eventually develop into a complete organism, specified by the information encoded in its DNA. It is important to realise that this specification is not sufficient for a complete description of the finished organism. Our understanding of nuclear transfer cloning illustrates the fact that DNA alone will not undergo the complex molecular processes associated with development, it must be placed in the context of a cell. Also, the DNA cannot be placed in any cell, it must be placed in an appropriate, as well as viable cell in order to begin replication. Thus, it is not possible to separate genetic content from its surrounds and retain any meaningful sense of a functional system. Computational models in, for example, the field of Artificial Life tend to emphasise DNA alone, and the systematic interdepencies of this process of development are not ususally considered. This tends to result in a model that is capable of exhibiting only very simplistic behaviour. A full model of such a process would have to be capable of exhibiting contextual responses like phenotypic plasticity where organisms with the same genotype may, if placed in a different environment, reveal significantly different phenotypes, to the extent that they may even be identified as different species [34].

Additional examples of high end complexity include:

1. The formation and modelling of ecosystems [8], where, for example, differing understandings of the same system can be generated from an analysis of it at varying time and spatial scales.

2. Language evolution and structure is difficult to correctly model, despite what is often a very detailed knowledge of its syntax. For example, the process whereby meaning is generated from initially meaningless utterances is very difficult to understand [35], and postmodern and deconstructive theories suggest that we should be careful in developing theories of meaning that are too simplistic [36].

3. The impact that culture has upon mental well-being appears to be significant. For example, culture mediates nearly every aspect of schizophrenea, from its identification and definition to symptom formation, and even to the eventual course and outcome of disorders [37]. This suggests that such conditions cannot be analysed reductively with an emphasis 
on biological factors or upon social factors alone. Rather, the complex interplay of these factors must be incorporated. This is a remarkably common problem, consider for example the nature versus nurture debate [38].

4. A more modern problem is the incorporation of a user into computing models, where different services might be requried by different users from what is in essence the same system. Often, solutions are too hardwired, and do not lend themselves to extension or adaption to new systems as they emerge.

It is interesting to notice that many of the systems exhibiting high end complexity have an evolutionary form. While we have a general understanding of the effect of the process of evolution in the biological realm, our formal and computational models of this process are sadly lacking. The fact that the more traditional modelling approaches have been unable to generate open ended evolution [2] suggests that our current methodologies are somehow flawed when it comes to the generation of interesting complex structure and behaviour. What is wrong with our models? A general model capable of generating novel hierarchical structure with different interactions and components at the different levels of understanding, as well as with a possibility for the open ended generation of emergent beaviour would go a long way to answering this question. Apparently the simple low level relational model of Process Physics offers a first step in that direction, but how does it differ from the more standard modelling methodologies?

A number of problems with our current modelling methodologies have been identified $[2,12]$ which, while not problematic when we consider some of the more traditional systems analysed by science, become almost impossible to circumvent when it comes to the modelling and the generation of systems exhibiting high end complexity. These include

A tendency to associate emergence with epistemology, or claim that new behaviour is only evident with respect to an observer. This generates a feeling among a number of complex systems science workers that emergence is not a phenomenon per se, rather that it exists only in the mind of an observer. Although there is undeniably an aspect of observer dependence in our modelling of high end complexity, this does not imply that the phenomenon of emergence is necessarily dependent upon the observer; our models might depend upon the observer, but the system itself may exhibit genuine emergence. For example, according to the most generally accepted theories of, for example, biological evolution, the Earth did not originally house life of any form, 
but gradually living forms arose, mutated and changed, forming new organisms, species, phylogenies etc. This is a process of the dynamical emergence of complex behaviour. While there are some borderline cases of species identification, it is generally agreed that species now exist which did not do so in the past, and this has a noticeable effect not just in our understanding of organisms, but upon the behaviour of the biotic components of the Earth's environment. Similar processes can be found in the evolution of linguistic ability, religion, and the generation of new technology. We require models capable of describing this dynamical process whereby new structures and interactions can come into existence.

The barrier of objects consists of the assumption that our models must necessarily describe things, which effectively rules out our ability to generate novelty. For example, in the standard agent based approaches there is a tendency to define an agent as an object capable of undergoing some number of interactions with its environment. However, this means that rather than generating novelty as agents move around some already defined environment, their modes of interaction are effectively used up, resulting in a point where no new behaviour is forthcoming.

The barrier of objectivity arises when we assume that objectivity is a core criterion of science despite a remarkable number of indications to the contrary which have been arising, often for centuries, in the generally 'softer' sciences, such as sociology, anthropology and history. Indeed, that last bastion of reductionism and objective measurements, physics, seems to be falling under a similar curse as quantum systems appear to be rather systematically revealing a tendency to reveal in different outcomes, each depending upon different choices of experimental arrangement.

All of these barriers are discussed more fully in a recent paper [12], which examines the way in which they impact upon our ability to understand and to generate high end complexity in our models, before proposing some possible resolutions to such barriers of modelling.

When we reconsider the model discussed in section 1.1, we see that it appears to be circumventing some of these problems: the Universe is taken to be real; a shift is attempted from more traditional object-based approaches to a more relational framework; and all analyses of higher level structures appear to be dependent in some way upon an observer (who sets thresholds in the low level relational model and then makes secondary models in order to characterise emergent behaviour within the system). However, none of these 
characteristics was actively sought when the initial model was investigated [39], rather they have been identified later when an attempt was made to understand the reasons for the success of the model [2]. Thus, we might ask at this stage how the historical basis of Process Physics differs from that of the more traditional reductive approaches. Can this basis form a foundation for a new modelling methodology capable of both modelling and generating complex emergent behaviour?

\section{The historical roots of Process Physics}

The fact that the very minimal relational system discussed in section 1.1 both exhibits observational emergence and generates a contextually dependent hierarchical structure suggests that it may have some sort of fundamental characteristic that could be used in a more general modelling of complex emergent behaviour. In order to fully explore this possibility we will look at the historical roots of the model.

The iterative map (1) was suggested by the Global Colour Model (GCM) of quark physics $[15,40]$. This model uses the functional integral method, and approximates low energy hadronic behaviour from the underlying QFT of quark-gluon behaviour, quantum chromodynamics (QCD). This section will discuss the way in which the iterative map (1) was extracted from from the GCM.

\subsection{The Global Colour Model (GCM) and the Func- tional Integral Calculus (FIC)}

QCD is the quantum field theoretic model of quarks and gluons. Starting from six quark fields, $\bar{q}$, six antiquark fields, $q$, and eight gluon fields, $A$, a classical action can be defined (in the Euclidean metric) as

$$
\begin{aligned}
S_{Q C D}\left[\bar{q}, q, A_{\mu}^{a}\right]=\int d^{4} x\left(\frac{1}{4} F_{\alpha \beta}^{a} F_{a}^{\alpha \beta}+\frac{1}{2 \xi}\left(\partial_{\mu} A_{\mu}^{a}\right)^{2}\right. & \\
& \left.+\bar{q}\left(\gamma_{\mu}\left(\partial_{\mu}-i g \frac{\lambda^{a}}{2} A_{\mu}^{a}\right)+\mathcal{M}\right) q\right)
\end{aligned}
$$

where $F_{\mu \nu}^{a}=\partial_{\mu} A_{\nu}^{a}-\partial_{\nu} A_{\mu}^{a}+g f^{a b c} A_{\mu}^{b} A_{\nu}^{c}$ describes the self-interaction of the gluons ( $f^{a b c}$ are the QCD structure constants, and $g$ is the colour charge), $\frac{\lambda^{a}}{2}$ are the eight $S U(3)$ colour generators in the Gell-Mann representation, $\gamma_{\mu}$ are the Dirac matrices, and $\mathcal{M}=\left\{m_{u}, m_{d}, \ldots\right\}$ are the quark current 
masses. This action can be quantized via a QCD generating functional

$$
Z=\int \mathcal{D} \bar{q} \mathcal{D} q \mathcal{D} A \exp \left(-S_{Q C D}[A, \bar{q}, q]\right)
$$

from which correlators of the general form

$$
\mathcal{G}(\ldots, x, \ldots)=\frac{\int \mathcal{D} q \mathcal{D} \bar{q} \mathcal{D} A \ldots x \ldots e^{-S_{Q C D}[A, \bar{q}, q]}}{\int \mathcal{D} q \mathcal{D} \bar{q} \mathcal{D} A e^{-S_{Q C D}[A, \bar{q}, q]}}
$$

can be extracted. These are then used to derive the predictions of QCD, however this proceedure is very complicated and for this reason a number of approximations have been developed.

The GCM $[15,40]$ is a low energy approximation which is used to extract the behaviour of hadrons from QCD. The model is motivated by the observation that at low energy (or long wavelength) we observe only those degrees of freedom associated with hadron behaviour; individual quarks and gluons are not directly observable. This suggests that a reasonable approximation to low energy behaviour could be achieved by choosing a set of variables which reflect only the behaviour that we can observe in an experimental setting. The choice of new variables is not arbitrary, it is achieved using what are termed Functional Integral Calculus techniques [41, 42, 43] which change variables in a dynamically determined way using analogues of the various techniques used in ordinary integral calculus. This process, which has been termed action sequencing [39] changes the variables of the QCD generating functional from the very simple expression in (10) to one that describes the hadron via a sequence of steps:

$$
\begin{aligned}
Z & =\int \mathcal{D} \bar{q} \mathcal{D} q \mathcal{D} A \exp \left(-S_{Q C D}[A, \bar{q}, q]\right) \quad(\mathrm{QCD}) \\
& \approx \int \mathcal{D} \bar{q} \mathcal{D} q \mathcal{D} A \exp \left(-S_{G C M}[A, \bar{q}, q]\right) \quad(\mathrm{GCM}) \\
& =\int \mathcal{D} B \mathcal{D} D \mathcal{D} D^{*} \exp \left(-S_{b l}\left[B, D, D^{*}\right]\right) \quad(\text { bilocal fields }) \\
& =\int \mathcal{D} \bar{N} \mathcal{D} N \ldots \mathcal{D} \pi \mathcal{D} \rho \mathcal{D} \omega . . \exp \left(-S_{\text {had }}[\bar{N}, N, . ., \pi, \rho, \omega, . .]\right)
\end{aligned}
$$

We might consider FIC as the first documented implementation of action sequencing. The details of this procedure are very complicated, the interested reader is referred to $[15,40]$ for further information. As an illustration of the power of this approach, we can examine the hadronic action obtained at the 
end of the complete action sequencing process (to low order) as,

$$
\begin{gathered}
S_{h a d}[\bar{N}, N, \ldots, \pi, \rho, \omega, \ldots]= \\
\int d^{4} x \operatorname{Tr}\left\{\bar{N}\left(\gamma . \partial+m_{0}+\Delta m_{0}-m_{0} \sqrt{2} i \gamma_{5} \pi^{a} T^{a}+\ldots\right) N\right\} \\
+\int d^{4} x\left[\frac{f_{\pi}^{2}}{2}\left[\left(\partial_{\mu} \pi\right)^{2}+m_{\pi}^{2} \pi^{2}\right]+\frac{f_{\rho}^{2}}{2}\left[-\rho_{\mu}\left(-\partial^{2}\right) \rho_{\mu}+\left(\partial_{\mu} \rho_{\mu}\right)^{2}+m_{\rho}^{2} \rho_{\mu}^{2}\right]\right. \\
+\frac{f_{\omega}^{2}}{2}[\rho \rightarrow \omega]-f_{\rho} f_{\pi}^{2} g_{\rho \pi \pi} \rho_{\mu} . \pi \times \partial_{\mu} \pi-i f_{\omega} f_{\pi}^{3} \epsilon_{\mu \nu \sigma \tau} \omega_{\mu} \partial_{\nu} \pi . \partial_{\sigma} \pi \times \partial_{\tau} \pi \\
-i f_{\omega} f_{\rho} f_{\pi} G_{\omega \rho \pi} \epsilon_{\mu \nu \sigma \tau} \omega_{\mu} \partial_{\nu} \rho_{\sigma} . \partial_{\tau} \pi \\
\left.+\frac{\lambda i}{80 \pi^{2}} \epsilon_{\mu \nu \sigma \tau} \operatorname{Tr}\left(\pi . F \partial_{\mu} \pi . F \partial_{\nu} \pi . F \partial_{\sigma} \pi . F \partial_{\tau} \pi . F\right)+\ldots\right] .
\end{gathered}
$$

We shall not list the new notation used in this action; details can be found in the review article [15] or $\mathrm{PhD}$ thesis [40]. However, even without the details of the notation, when we compare this expression to that of the QCD action (9) we see a vast difference in the complexity of the two equations. Equation (16), which is is only a low order expansion suggests that there is a very rich set of behaviour evident in the behaviour of the nucleon; it is an extremely complex system, but its description is well approximated by this theory. Thus, some very complicated emergent behaviour has been extracted using this technique. Is it possible that this technique could be generalised in some way? A first step towards answering this question was taken when the iterative equation (1) was extracted from this model.

\subsection{The Extraction of the Iterator Equation}

The richness of the behaviour exhibited by this model of hadrons led to the hypothesis that it may be possible to regain much of the dynamics in physics without incorporating it into our models axiomatically. We take as our starting point an action that arises in the action sequencing of the generating functional (in equation (14)). This action describes the dynamics of bilocal fields $B$ (which can be considered very similar to the connections 
between nodes in (1)) and appeared in an early ${ }^{6}$ GCM paper [41]

$$
S[B]=-\operatorname{Tr} \operatorname{Ln}\left[\not \partial \delta(x-y)+\frac{M^{\theta}}{2} B^{\theta}(x-y)\right]+\int d^{4} x d^{4} y \frac{B(x, y) B(y, x)}{2 g^{2} D(x-y)},
$$

where $\not \partial(x-y)$ represents an essentially localised momentum term, the $M^{\theta}$ term is due to a bilocal meson field, $B^{\theta}(x-y)$ is a hermitian bilocal field and $D(x-y)$ is a complex bilocal field. See the early paper for details, or the more modern and very comprehensive $\mathrm{PhD}$ thesis [40].

The stochastic quantisation (SQ) procedure of Parisi and $\mathrm{Wu}[44,45]$ is used to quantize this action. SQ consists of introducing a 5th time $\tau$ in addition to the usual 4 space-time points $x_{\mu}$ and postulating that the dynamics of some field represented by $\phi$ in this extra time $\tau$ is given by the Langevin equation:

$$
\frac{\partial \phi(x, \tau)}{\partial \tau}=-\frac{\delta S[\phi]}{\delta \phi}+\eta(x, \tau)
$$

where $\eta$ is a Gaussian random variable. The stochastic average of all fields $\phi_{n}$ satisfying equation (18) is calculated where $\tau_{1}=\tau_{2}=\cdots=\tau_{l}$, and finally the limit $\tau_{1} \rightarrow \infty$ is taken. Parisi and Wu proved perturbatively that in this limit the average is equal to the correlation function of the field of interest, i.e., an $l$-point correlation can be defined where

$$
\lim _{\tau_{1} \rightarrow \infty}\left\langle\phi_{\eta}\left(x_{1}, \tau_{1}\right) \phi_{\eta}\left(x_{2}, \tau_{1}\right) \cdots \phi_{\eta}\left(x_{l}, \tau_{1}\right)\right\rangle_{\eta}=\frac{\int \mathcal{D} \phi \phi\left(x_{1}\right) \phi\left(x_{2}\right) \ldots \phi\left(x_{l}\right) e^{-S[\phi]}}{\int \mathcal{D} \phi e^{-S[\phi]}} .
$$

Thus, at least in the perturbative sense, SQ provides an alternative route to quantization, and in particular to the correlation functions of QFT.

We make use of this procedure to quantize the action (17), substituting it into the Langevin equation (18) to obtain

$$
\frac{\partial B\left(x^{\mu}, \tau\right)}{\partial \tau}=-\frac{\delta S[B]}{\delta B}+\eta\left(x^{\mu}, \tau\right)
$$

\footnotetext{
${ }^{6}$ This paper was an early attempt at using FIC to bosonize QCD but made use of a less appropriate change of variables than the later work utilizing this technique. The change of variables used here was to $\mathbf{1}_{c}$ and $\mathbf{8}_{c}$ bilocal $\bar{q} q$ variables which worked well in the extraction of meson observables but was less physical, due to the $\boldsymbol{8}_{c}$ fields which are repulsive for for $\bar{q} q$ states. The later work was based upon $\mathbf{1}_{c}$ meson variables coupled with $\overline{\mathbf{3}}_{c}$ and $\mathbf{3}_{c}$ diquark variables [42]. The less physical equation was used as a basis for the derivation because it is slightly simpler than its equivalent in the later work but still results in rich behaviour.
} 
Thus, the $B$ field will dynamically update in this time parameter as $B \rightarrow$ $B+\delta B$, that is:

$$
B \longrightarrow B-\frac{\delta S[B]}{\delta B}+\eta\left(x^{\mu}, \tau\right)
$$

Returning to the action (17), we make a number of simplifications which strip away structure considered unimportant to the model. Namely we set any variables that are deemed irrelevant equal to 1 , drop the gluon interaction term $D(x-y)$ and set the variables of the $B$ terms in the integral term as equivalent (i.e., assuming no significant interaction between the fields at this level of the description). The term $\not \partial \delta(x-y)$ represents an essentially localised momentum term and is ignored. Similarly the trace operation is discarded as it simplifies too much of the behaviour of a system that has already been dramatically simplified. This results in a simpler action:

$$
S[B]=\operatorname{Ln}[B]+\int d^{4} x d^{4} y B(x, y)^{2} .
$$

Now, making a transition to a matrix based lattice representation in place of the continuous representation above, we obtain

$$
S\left[B_{i j}\right]=B_{i j}^{2}+\operatorname{Ln}\left[B_{i j}\right] .
$$

substituting (23) into (21), and changing the symbol $\eta$ to $\omega$, an equivalent noise term which operates over the matrix representation of the $B$ field results in an update equation:

$$
B_{i j} \rightarrow B_{i j}-\left(\frac{\delta}{\delta B_{i j}} B_{i j}^{2}+\frac{\delta}{\delta B_{i j}} \operatorname{Ln}\left[B_{i j}\right]\right)+\omega_{i j}
$$

Finally, we make use of the calculus identities $\delta B^{2} \rightarrow B$ and $\delta \ln B \rightarrow B^{-1}$ to extract the structure of the desired equation (1).

It is worth emphasising that the use of the stochastic quantization procedure in this process suggests that a system emergent from this equation should exhibit quantum behaviour in the limit of sufficient numerical experiments that run for a long enough time. Note also that this is more of an extraction than a derivation; there is almost a sense of observation in the derivation itself, where we choose which variables to consider relevant and which to ignore.

It is remarkable how much richness of behaviour is retained in the behaviour of (1), this is despite the dramatic loss of standard physical structure that is characteristic in this derivation. In this case it appears that a set of key characteristics are driving this behaviour. Some of these include the 
nonlinear aspect of the equation and its grossly 'nonlocal' and holistic form, as well as the noise term that pushes its behaviour away from equilibrium. Most important perhaps is the structure of the general equation, it is close enough to the bilocal action that it incorporates its key physical properties in some way.

Indeed, it has been argued [2] that the key structure remaining after this extraction is related to the Nambu-Goldstone (NG) modes [30] that are used in the action sequencing of the QCD action. Indeed, the NG-modes form the basis of the choice of physical variables in this process [15]. The GCM is particularly effective in revealing the NG phenomena that follows from the dynamical breaking of chiral symmetry [30]. Indeed, the GCM results in the complete derivation of the Chiral Perturbation Theory phenomenology, but with the added feature that the induced NG effective action is non-local, so that the usual non-renormalisability problems do not arise. It is highly likely that NG-modes are particularly important in the modelling of complex emergent behaviour [2], a point that we shall return to shortly.

It is expected that there will be a class of equations all of which exhibit behaviour similar to that discussed in section 1.1, but at this point in time (1) is the only one known. There is some reason to suppose that such a class of models is already being discovered in the field of complex systems science, consider for example a very simple network model on the rise and fall of societies [46] that behaves very similarly to (1). Is it possible that a class of equations such as (1) can be used in the modelling of complex emergent behaviour? What can be determined about such a general class of models?

\section{Quantum Theories as Models of Complex- ity}

It is very likely that an essential characteristic of such a class of models will lie in in quantum theory. This section will briefly discuss this link between quantum theories and models of complex behaviour, a more in-depth discussion is currently in preparation.

All quantum theories have the same fundamental structure. Indeed the implementation of any quantum theory involves following the same general recipe:

1. First, a map from a classical state space, $S$ to a complex number $\mathbf{C}$ is found. This map is often written in the form of the symbol $\psi$.

2. Depending upon the system under examination some time evolution equation is chosen from a set of possibilities including the Schrödinger 
equation, the Klein-Gordon equation, the Dirac equation etc., each of which map to another set of complex numbers.

3. Steps 1 and 2 are mathematically well defined and understood. However, complex numbers are never revealed when a measurement is performed on a quantum system. Instead, the system is found to be in some classical state, related to the configuration of the experiment performed $[47,48]$. The dynamics of this process are not understood, and there are a number of competing theories of quantum measurement, each of which lead to a different interpretation of quantum mechanics $[47,49]$. Mathematically, the process of measurement is implemented by mapping the inner product of $\psi$ at the point of time in which we are interested to some sort of probability space from which a set of predictions about the system are obtained, in a basis determined by the act of measurement itself.

Even the implementation of QFT's involves the same proceedure, albeit in a far more complex form [2], a point that becomes obvious with a shift to the modern path integral formulation [50].

Although traditionally the quantum formalism has only been applied to a very particular set of systems, a wide variety of more novel applications are starting to appear, where quantum theories of macroscopic systems are being created, often quite successfully [51]. For example, different varieties of the quantum formalism have been applied to situations such as: stock market analysis [52]; quantum models of the brain [53, 54]; models of cognitive function and concepts $[55,56,57]$; modelling of the process of decision making in situations of ambiguity [58] etc. This general use (some might argue abuse) of the quantum formalism suggests that it is indeed far more generally applicable than is traditionally considered to be the case, and indeed the above consideration of the form of the quantum formalism suggests a reason for this; there is no mention of macroscopic detectors or microscopic particles in this formalism, and there is no reason to supose a priori that they are necessary. This is merely an historical bias resulting from the discovery of the quantum formalism as a description of a specific class of systems (i.e., microscopic ones).

A clue to this apparent generality of the quantum formalism lies in the theorems, generally attributed to Bell, of nonlocality and contextuality [48]. Each of these theorems rely upon showing that when a quantum system is entangled ${ }^{7}$ there exists a set of observables for which it is impossible to

\footnotetext{
${ }^{7}$ An entangled state consists of at least two noninteracting systems $A$ and $B$, represented by the states $\psi_{A}$ and $\phi_{B}$ on the Hilbert spaces $H_{A}$ and $H_{B}$, the composite state of
} 
consistently assign an eigenvalue i.e., the outcomes of measurements of apparently independent experiments are incompatible. The resolution to this incompatibility lies in a proper consideration of the experimental arrangement; performing one experiment always results in a change of the quantum system and rules out the possibility of performing an alternative one. Thus, it is impossible to completely describe a quantum system without reference to its context. Entangled quantum systems exhibit a form of nonseparable behaviour and should not be considered independently of the set of measurements performed upon them.

This situation shares much similarity with systems exhibiting high end complexity. Such systems should not be considered independently of their context, and may show incompatible results depending upon the measurements to which they are subjected. For example, as was discussed in section 3 the social context in which schizophrenia occurs can have a dramatic effect upon the course of a patients illness. Indeed, different patients may be classified as schizophrenic or not depending upon the culture in which they are being diagnosed [37]. This situation is analagous to the incompatible measurements occuring in the quantum formalism, and hence it is expected that the very well developed quantum formalism could be used to provide models of such contextual dependency during measurement.

This suggests that the quantum theoretic formalism can be understood as modelling generic situations of contextuality where a system cannot be considered reductively as a set of separable subcomponents uninfluenced by their environment, even in cases where two subsystems are spread over a distance [2]. This contextual dependence often manifests itself as randomness arising from a lack of knowledge about the outcome of experiments, which can be used to explain the appearance of randomness in systems exhibiting contextual behaviour, including quantum ones [59].

Two specific examples of the way in which the quantum formalism could be used to model such systems can be found quite quickly. Firstly, it is possible to formulate Bell-type inequalities which can be used to test whether a system is exhibiting contextual responses [12]. Another possibility would consist of incorporating the effect that an observer might have upon a system through finding a set of basis states to represent the system that are compatible with the basis states available to an observer and then performing 'measurements' as is done in standard quantum theory [60].

If QT is capable of describing such a rich set of behaviour then we might ask what is to be gained by a QFT as opposed to the simpler QT. The answer

which is written $\psi_{A} \otimes \phi_{B}$ which cannot itself be separated in a meaningful manner as a product $\psi_{A} \phi_{B}$. 
to this question lies at the heart of complex emergent behaviour. Some clues are supplied by early work performed by pioneers such as Primas who noted that it is possible to perform a perturbation expansion over multiple time scales [61], by Froölich who proposed that coherent phase correlations will play "a decisive role in the description of biological materials and their activity" [62], and Davydov who proposed the concept of a biological soliton that resists thermal fluctuations [63]. Taken together these results suggest that QFT has many applications in nontraditional fields, but despite this initial promise there has been a tendency for the larger physics community to ignore these possible extra applications.

The full power of QFT becomes evident when symmetry breaking is incorporated into the formalism. If a system falls into a situation where its ground state does not have the same continuous symmetry as its dynamics then it is considered to be exhibiting spontaneous symmetry breaking. According to Goldstone's theorem, a system in such a state will dynamically generate massless bosons, termed Nambu-Goldstone modes (NG-modes), in response to this break in symmetry. The number of NG-modes generated will equal the number of broken symmetries in the system [30]. Due to their massless nature, NG-modes are long-range, they can move through an entire system with no loss of energy, providing it with long range coherence to such systems [54]. Because of their boson status many NG-modes may occupy the same ground state without changing the energy of the system. In such systems there can be a number of structurally different ground states, each in a lowest energy configuration. This is not possible in a classical system; there is only one lowest energy state in the classical realm, which means that there is only one way in which a system can exhibit stable behaviour. On the other hand systems described by a QFT can exist in many different stable configurations, which allows them to change state from one stable ground state to another and in the process generate new behaviour of a very rich form. Because of this phenomenon, QFT is the only quantum formalism capable of generating truly emergent behaviour rather than merely modelling a set of components and their interactions $[2,54,64]$. This is to be expected since its basis could be argued to lie in the necessity of modelling the creation and annihilation of particles in modern high energy experiments; QFT was invented in order to model the emergence of new particles.

Thus, the origin of Process Physics in QFT and, in particular its dependence upon NG phenomena via the extraction of (1) from the GCM suggests that such a model can, not only exhibit contextual responses, but can truly generate complex emergent behaviour. 


\section{Generating Complex Emergent Behaviour - a new modelling approach?}

We shall conclude with a brief summary of some of the more recent developments and applications that have followed from this more process driven approach to modelling complex emergent behaviour.

The historical basis of Process Physics in QFT suggests that such theories are capable of describing far more systems than has generally been considered the case. As described above, the process of action sequencing applied in the extraction of hadronic structure from the GCM relies strongly upon the use of symmetry breaking and the associated emergence of Nambu-Goldstone modes which are chosen as the dynamically extracted new variables. It is expected that this process will provide the key to the discovery of a general mechanism whereby complex emergent behaviour can be generated [2] This idea has been explored [2, 12] using a very simple model of sympatric speciation which is based upon quantum field theory. The model utilises dynamical symmetry breaking and the associated emergence of NG-modes to model the emergence of new sets of species within a system undergoing dynamical change. This model of speciation can be considered as a model of the differentiation of a species, and it is expected that such models can be generalised to a model of the process of differentiation as it occurs in many systems exhibiting this form of behaviour. For example, a key future goal is to use these concepts to create a model of the process of biological development. This process can be understood as consisting of two sub-processes, differentiation (the specification of different cell fates) and morphogenesis (the development of shape), and leads to the formation of an organism. It is expected that far more applications are possible. This is because differentiation can be understood as the process responsible for forming new word meanings in language, new religions, new cultures and societies etc.. Hence, a dynamical model of differentiation could be broadly used in modelling processes of generation, evolution and the creation of novelty. A general class of such models would be very important in the modelling of systems exhibiting high end complexity.

Interestingly, some models along these lines already exist. For example, a quantum field theoretic model of the brain has been developed over a number of years, which makes use of the infinite number of unitarily inequivalent ground states required by QFT to model processes such as the formation of long term memories as situations where new stable states describing the brain are created by the exposure of a person to new ideas etc. Short term memories are described as excitations of the ground state, and the process of 
recall is modelled using the excitation of NG-modes from the current ground state of the brain. This model explains the serial nature of association as a situation where an excitation gradually decays via intermediate states back into a ground state. The book by Vitiello [54] contains a good overview of this work, as well as an exhaustive list of references to the more detailed papers describing these results. Thus, there is every reason to suppose that such models could be very useful in the dynamical modelling of emergent stable structures in general. Indeed, in the pioneering paper on the QFT brain model [65], Ricciardi and Umezawa suggest that a similar model might possibly be used to explain how a stable DNA code is dynamically generated.

Another promising avenue for the quantum modelling of complexity lies in the modelling of human interactions with social, computational and ecological systems providing services to those humans. With the generalised understanding of quantum theories summarised in section 5, we begin to understand something that has been appreciated by the socalled 'soft' sciences almost since their inception; that contradictory results can be obtained from the assumption that different choices of experiment are independent from one another. However, with an acceptance that contextual outcomes are the norm of the quantum formalism we acquire a new, less arbitrary, theory with which we can incorporate choice into scientific discourse. For example, the concept of a "service ecosystem" has recently been developed [66] to describe the connection of different web services which are deployed, published, discovered and delivered to different business channels through specialist intermediaries. In such cases a richer range of semantics in service descriptions and support of fuzzier search goals becomes necessary in order to create adequate descriptions and results for the end users. Such systems are highly contextual, and as such, their modelling by the standard reductionist methodology is inappropriate. However, with a formalism that incorporates contextual outcomes into its very foundation, quantum theory provides a number of possibilities for a set of more appropriate modelling techniques. Instead of hardwiring a plethora of different services into any attempt to describe such systems, a more malleable and hence long term solution presents itself along the lines discussed in section 5 [60]. Namely the effect of the user upon a system can be modelled through finding a set of basis states to represent the system that are compatible with the basis states available to functions performed by a user and then performing 'measurements' as is done in standard quantum theory Thus, we see a general possibility that quantum theories can provide a new class of models capable of incorporating more fully the choices made by an observer who takes a more active role in the behaviour of a system than is traditionally considered to be the case, i.e., is interacting with the system in choosing which measurements will be performed upon it. 
Finally, the simple relational model discussed in section 1.1 has been particularly successful as a pregeometric theory. It has been used as the basis of a higher level theory of quantum gravity [1] which is proving remarkably successful in the explanation of a number of so far unexplained phenomena. However this theory is remarkably complex when compared to the more traditional object based approaches. This leads to the final question of this paper and indeed to our conclusions.

\section{What has been gained by a shift to Process Physics? (In Conclusion)}

The shift to a process oriented physics is remarkably difficult to implement. All of our standard modes of analysis are based upon reductive, object based techniques that assume a passive observer unable to interact with the system of interest in any way other than via controllable, identified channels. In attempting to construct a more complex understanding of the world (which is in fact more complex) we lose many of the long held, almost sacrosanct, assumptions of the scientific method. The accompanying difficulty of analysis is to be expected. However, many of the phenomena and problems currently unexplainable, and even impossible to correctly frame in more standard approaches become at least approachable from this new perspective. Many mysteries of physics become less mysterious, and as this paper has attempted to show, some of the problems outside of standard physics also appear to be amenable to this new methodology. It has been argued in this paper that the success of such models will lie with the general class of quantum theories, and in particular, in the class of QFT's.

In conclusion I would like to point out that I still teach the standard object-driven and static physics to undergraduates, in much the same way that I begin a discussion of mechanics with Newton's laws rather than with General Relativity. There is a very good reason for this, and it is not merely historical. Taking account of complexity is a very difficult process, and in many scenarios it is not necessary. Even in situations where context must be incorporated it is not always necessary, or even desirable to go to a fullyfledged QFT, and it is highly likely that there will be a large number of complex systems that will not be describable by any form of quantum formalism. Systems exhibiting high end complexity cannot be fully modelled by one language of description alone $[2,12]$; we require more tools and more approaches before we can ever hope to understand this very important class of system, and the approach outlined above is merely one proposal in this 
direction.

The author would like to acknowledge the support of her PhD supervisor R.T. Cahill, without whose support she could have taken a lifetime to generate these concepts.

\section{References}

[1] R. T. Cahill. Process Physics: From Information Theory to Quantum Space and Matter. Nova Science Publishers, 2005.

[2] Kirsty Kitto. Modelling and Generating Complex Emergent Behaviour. PhD thesis, School of Chemistry Physics and Earth Sciences, The Flinders University of South Australia, 2006.

[3] W. Ross Ashby. An Introduction to Cybernetics. Chapman and Hall, London, 1956.

[4] Robert Rosen. Essays on Life Itself. Complexity in Ecological Systems Series. Columbia University Press, New York, 2000.

[5] J. Horgan. From complexity to perplexity. Scientific American, 272:7479, 1995.

[6] Kirsty Kitto. Dynamical Hierarchies in Fundamental Physics. In Bilotta et al. [67], pages 55-62.

[7] Stanley N. Salthe. Evolving Hierarchical Systems: Their Structure and Representation. Columbia University Press, New York, 1985.

[8] R. V. O'Neill, D. L. DeAngelis, J. B. Waide, and T. F. H. Allen. A Hierarchical Concept of Ecosystems, volume 23 of Monographs in Population Biology. Princeton University Press, Princeton, New Jersey, 1986.

[9] D.T. Campbell. 'Downward causation' in hierarchically organized biological systems. In F.J. Ayala and T. Dobzhansky, editors, Studies in the Philosophy of Biology, pages 179-186. Macmillan Press, London, 1974.

[10] G. Nicholis and I. Prigogine. Self-Organization in Non-Equilibrium Systems: From Dissipative Structures to Order through Fluctuations. J. Wiley and Sons., 1997. 
[11] A.S. Davydov. Biology and quantum mechanics. Pergamon, Oxford, 1982.

[12] Kirsty Kitto. High End Complexity. International Journal of General Systems, 2007. In Press.

[13] Per Bak. How Nature Works: The Science of Self-Organized Criticality. Springer-Verlag, New York, 1996.

[14] P. Bak, C. Tang, and K. Wiesenfeld. Self-organized criticality. Physical Review A, 38:364-374, 1988.

[15] Reginald T. Cahill and Susan M. Gunner. The global colour model of QCD for Hadronic processes — a review. Fizika, B7:171-202, 1998.

[16] C. M Klinger. Process Physics: Bootstrapping Reality fom the Limitations to Logic. PhD thesis, School of Chemistry Physics and Earth Sciences, The Flinders University of South Australia, 2005.

[17] G. Nagels. Space as a "bucket of dust". General Relativity and Gravitation, 17(6):545-557, 1985.

[18] R.T. Cahill and C.M. Klinger. Self-Referential Noise as a Fundamental Aspect of Reality. In D. Abbott and L. Kish, editors, Proceedings of the 2nd International Conference on Unsolved Problems of Noise and Fluctuations (UPoN'99), volume 511, page 43, New York, 2000. American Institute of Physics.

[19] S. N. Salthe. Summary of the principles of hierarchy theory. General Systems Bulletin, 31:13-17, 2002.

[20] Nils A. Baas. Emergence, hierarchies, and hyperstructures. In C. Langton, editor, Artificial life III, pages 515-537. Addison-Wesley, Advanced Book Program, Santa Fe, New Mexico, 1994.

[21] Howard H. Pattee. The Physical Basis and Origin of Hierarchical Control. In Hierarchy Theory: The challenge of complex systems, pages 71-156. George Braziller Inc., 1973.

[22] Herbert A. Simon. The Sciences of the Artificial. MIT Press, Cambridge, Massachusetts, 3 edition, 1996.

[23] Tom Lenaerts, Dominique Groß, and Richard Watson. On the modelling of dynamical hierarchies: Introduction to the workshop WDH 2002. In Bilotta et al. [67], pages 37-44. 
[24] George Kampis. The Inside and Outside Views of Life. In F. Moran, A. Moreno, J.J. Merelo, and P. Chacon, editors, Advances in Artificial Life, Third European Conference on Artificial Life Granada, Spain, June 4-6, 1995, pages 95-102. Springer, 1995.

[25] George Kampis. Self-Modifying systems in biology and cognitive science. Pergamon Press Inc, Oxford, 1991.

[26] Francisco Varela and Jonathan Shear, editors. The View from Within: First-person approaches to the study of consciousness, volume 6 of Journal of Consciousness Studies. Imprint Academic, Thorverton, UK, 1999.

[27] O.E. Rössler. Endophysics. In J. Casti and A. Karlquist, editors, Real Brains - Artificial Minds. North-Holland, New York, 1987.

[28] D. Finkelstein and S.R. Finkelstein. Computer Interactivity Simulates Quantum Complementarity. International Journal of Theoretical Physics, 22:753-779, 1983.

[29] R. T. Cahill. Process Physics: From Information Theory to Quantum Space and Matter. Process Studies Supplement, 5:1-131, 2003.

[30] Robert E. Marshak. Conceptual Foundations of Modern Particle Physics. World Scientific, Singapore, 1993.

[31] J.-J. Dugne, S. Fredriksson, and J. Hansson. Preon trinity — a schematic model of leptons, quarks and heavy vector bosons. Europhysics Letters, 60(2):188-194, 2002.

[32] Mark A. Bedau, John S. McCaskill, Norman H. Packard, Steen Rasmussen, Chris Adami, David G. Green, Takashi Ikegami, Kinihiko Kaneko, and Thomas S. Ray. Open problems in Artificial Life. Artificial Life, 6:363-376, 2000.

[33] Bruce Edmonds. Syntactic Measures of Complexity. PhD thesis, University of Manchester, 1999.

[34] Mary Jane West-Eberhard. Phenotypic Plasticity and the Origins of Diversity. Annual Review of Ecology and Systematics, 20:249-278, 1989.

[35] John Maynard Smith and Eörs Szathmáry. The Major Transitions in Evolution. Oxford University Press, Oxford, 1997.

[36] J. Derrida. Différance. In Margins Of Philosophy, pages 3-27. University of Chicago Press, Chicago, 1982. 
[37] Janis Hunter Jenkins and Robert John Barrett. Schizophrenia, Culture, and Subjectivity: The Edge of Experience. Cambridge University Press, New York, 2004.

[38] Steven Pinker. The Blank Slate: The Modern Denial of Human Nature. Viking Penguin, London, 2002.

[39] R. T. Cahill and C. M. Klinger. Pregeometric Model of the Spacetime phenomenology. Physics Letters, A223:313-319, 1998.

[40] Susan M. Gunner. Modelling Low Energy Processes in Quantum Chromodynamics using the Global Colour Model. PhD thesis, School of Chemistry Physics and Earth Sciences, The Flinders University of South Australia, 2004.

[41] R. T. Cahill and C. D. Roberts. Soliton bag models of hadrons from QCD. Physical Review D, 32(8):2419-2428, 1985.

[42] R. T. Cahill. Hadronisation of QCD. Australian Journal of Physics, 42:171-186, 1989.

[43] R. T. Cahill, J. Praschifka, and C. J. Burden. Diquarks and the bosonisation of QCD. Australian Journal of Physics, 42:161-169, 1989.

[44] G. Parisi and Yongshi Wu. Perturbation theory without gauge fixing. Scientia Sinica, 24:483-490, 1981.

[45] E. Gozzi. Functional-integral approach to Parisi-Wu stochastic quantization: scalar theory. Physical Review D, 28(8):1922-1930, 1983.

[46] Markus Brede and John Finnigan. On the Rise and Fall of Complex Societies. http://www.dar.csiro.au/css/August Workshop 06.shtm, 2006. Talk presented at Complex Systems in Focus CSIRO CSS Annual Workshop Aug 8-10 Coogee Bay Hotel, Sydney.

[47] F. Laloë. Do we really understand quantum mechanics? Strange correlations, paradoxes, and theorems. American Journal of Physics, 69(6):655-701, 2001.

[48] N. David Mermin. Hidden variables and the two theorems of John Bell. Reviews of Modern Physics, 65(3):803-815, 1993.

[49] John S. Bell. Six possible worlds of quantum mechanics. pages 181-195. Cambridge University Press, Cambridge, 1987. 
[50] Michio Kaku. Quantum Field Theory: a modern introduction. Oxford University Press, Oxford, 1993.

[51] P. D. Bruza, W. Lawless, C.J. van Rijsbergen, and D. Sofge, editors. Proceedings of the AAAI Spring Symposium on Quantum Interaction. March 27-29, Stanford University, 2007. AAAI Press.

[52] Belal E. Baaquie. Quantum Finance: Path Integrals and Hamiltonians for Options and Interest Rates. Cambridge University Press, 2004.

[53] Roger Penrose. The emperor's new mind. Penguin, New York, 1991.

[54] Giuseppe Vitiello. My Double Unveiled. John Benjamins Publishing Company, Amsterdam, 2001.

[55] D. Aerts and L. Gabora. A theory of concepts and their combinations I: the structure of the sets of contexts and properties. Kybernetes, 34:151$175,2005$.

[56] L. Gabora and D. Aerts. Contextualizing Concepts using a Mathematical Generalization of the Quantum Formalism. Journal of Experimental and Theoretical Artificial Intelligence, 14:327-358, 2002.

[57] P.D. Bruza and R.J. Cole. Quantum Logic of Semantic Space: An Exploratory Investigation of Context Effects in Practical Reasoning. In S. Artemov, H. Barringer, A.S. d'Avila Garcez, L.C. Lamb, and J. Woods, editors, We Will Show Them: Essays in Honour of Dov Gabbay, volume 1, pages 339-361. College Publications, 2005.

[58] D. Aerts. Towards a new democracy: Consensus through quantum parliament. In D. Aerts, B. D'Hooghe, and N. Note, editors, Worldviews, Science and Us, Redemarcating Knowledge and its Social and Ethical Implications. World Scientific, Singapore, 2005.

[59] D. Aerts. The hidden measurement formalism: what can be explained and where paradoxes remain. International Journal of Theoretical Physics, 37:291-304, 1998.

[60] P.D. Bruza, K. Kitto, and A. Barros. Exploration of non-reductionist models of service ecosystems. In preparation. Abstract accepted at Complex'07 - The 8th Asia-Pacific Complex Systems Conference, 2007.

[61] Hans Primas. Chemistry, Quantum Mechanics and Reductionism. Perspectives in Theoretical Chemistry. Springer-Verlag, Berlin, 2 edition, 1983. 
[62] H. Fröhlich. Theoretical physics and biology. In H. Fröhlich, editor, Biological coherence and response to external stimuli, pages 1-24. SpringerVerlag, Berlin, 1988.

[63] A.S. Davydov. Solitons in molecular systems. Kluwer, Dordrecht, 1991.

[64] Hiroomi Umezawa. Advanced Field Theory: Micro, macro, and thermal physics. American Institute of Physics, New York, 1993.

[65] L.M. Ricciardi and H. Umezawa. Brain and Physics of Many-Body Problems. Kibernetik, 4:44-48, 1967.

[66] Alistair Barros, Marlon Dumas, and Peter Bruza. The Move to Web Service Ecosystems. BP Trends, 3(12), 2005.

[67] Eleonora Bilotta, Dominique Groß, Tom Smith, Tom Lenaerts, Seth Bullock, Henrik Hautop Lund, Jon Bird, Richard Watson, Pietro Pantano, Luigi Pagliarini, Hussein Abbass, Russell Standish, and Mark Bedau, editors. ALife VIII - workshops. University of New South Wales, 2002 . 